\title{
AVE 0991 attenuates cardiac hypertrophy through reducing
}

\section{oxidative stress}

Yuedong Ma ${ }^{\mathrm{a}, 1}$, Huiling Huang ${ }^{\mathrm{a}, 1}$, Jingzhou Jiang ${ }^{\mathrm{a}, 1}$, Lingling $\mathrm{Wu}^{\mathrm{a}}$, Chunxi Lin ${ }^{\mathrm{a}}$, Anli Tang ${ }^{\text {a }}$, Gang Dai ${ }^{\text {a }}$, Jiangui He ${ }^{\text {a,* }}$, Yili Chen ${ }^{\text {a,* }}$

${ }^{a}$ Department of Cardiology, the First Affiliated hospital of Sun Yat-Sen University and Key Laboratory on Assisted Circulation, Ministry of Health, Guangzhou 510080, P.R.China

${ }^{1}$ The authors contributed equally to this article and should be considered equal first authors.

${ }^{2}$ The authors contributed equally to this article and should be considered equal corresponding author

Short title: AVE 0991 attenuates cardiac hypertrophy

*Corresponding author:

Yili Chen and Jiangui He

Department of Cardiology

The First Affiliated Hospital of Sun Yat-Sen University

58 Zhongshan II Road

Guangzhou, P.R. China, 510080

Email: 3160992650@qq.com; hejiangui@163.com

Tel: (86) 20 87332200-8151 Fax: (86) 2087330939 


\section{Abstract}

AVE 0991, the nonpeptide angiotensin-(1-7) (Ang-(1-7)) analog, is recognized as having beneficial cardiovascular effects. However, the mechanisms have not been fully elucidated. This study was designed to investigate the effects of AVE 0991 on cardiac hypertrophy and the mechanisms involved. Mice were underwent aortic banding to induce cardiac hypertrophy followed by the administration of AVE 0991 (20 mg.kg·day(-1)) for 4 weeks. It was shown that AVE 0991 reduced left ventricular hypertrophy and improved heart function, characterized by decreases in left ventricular weight and left ventricular end-diastolic diameter, and increases in ejection fraction. Moreover, AVE 0991 significantly down-regulated mean myocyte diameter and attenuate the gene expression of the hypertrophic markers. Furthermore, AVE 0991 inhibited the expression of NOX 2 and NOX 4, meaning that AVE 0991 reduced oxidative stress of cardiac hypertrophy mice. Our data showed that AVE 0991 treatment could attenuate cardiac hypertrophy and improve heart function, which may be due to reduce oxidative stress.

Key words: AVE 0991; cardiac hypertrophy; oxidative stress; pressure overload 


\section{Introduction}

Cardiac hypertrophy is an adaptive response to physiological and pathological overload. It is beneficial in reducing stress placed on the heart. However, when the stress is sustained, it becomes pathological and leads to cardiac dysfunction and heart failure [1-3]. Moreover, recent evidence demonstrated that oxidative stress was involved in the pathophysiology of cardiac hypertrophy and congestive heart failure. Oxidative stress describes an imbalance between antioxidant defences and the production of reactive oxygen species (ROS), which at high levels cause cell damage but at low levels lead to subtle changes in intracellular pathways [4-6]. ROS induce matrix metalloproteinase (MMP) activity that mediates cardiac hypertrophy [4].

The nonpeptide Ang-(1-7) analog, AVE 0991, is recognized as having beneficial cardiovascular effects similar to those induced by Ang-(1-7) [7-11]. Recently, Ferreira et al. described AVE 0991 treatment attenuated isoproterenol-induced impairment of heart function and remodeling [8]. Other studies have demonstrated AVE 0991 restore endothelium-dependent vascular relaxation by down-regulating vascular oxidant stress and enhancing NO availability [12]. Our studies have shown that AVE 0991 treatment ameliorates cardiomyocyte hypertrophy via down-regulation of transforming growth factor (TGF)- $\beta 1 /$ Smad 2 signaling [7]. However, little is known about the effects of AVE 0991 treatment in cardiac hypertrophy induced by pressure overload and the mechanisms.

In the present study, we used a rat model of aortic banding which induce pressure overload to determine whether AVE 0991 treatment influences cardiac hypertrophy 
and function, and determine whether oxidative stress are involved in AVE 0991-mediated effect.

\section{Materials and methods}

The investigation was carried out according to the Guide for Care and Use of Laboratory Animals published by US National Institute of Health (NIH Publication No. 85-23, revised 1996).

\subsection{Animals}

Male C57BL/6J mice (8-10 weeks old; 24-26 g) were obtained from the Experimental Animal Facility of Sun Yat-sen University, China. All animals were housed in individual cages on a $12 \mathrm{~h}$ light-dark cycle in a room with temperature $\left(24 \pm 2^{\circ} \mathrm{C}\right)$ and humidity control, and with ad libitum access to tap water and standard rodent chow. Mice were anesthetized by intraperitoneal injection of $1.5 \%$ pentobarbital $\left(\mathrm{W}^{*} 0.06\right)$ and cardiac hypertrophy was induced by pressure overload, which was performed by descending aortic banding (AB). Similar surgery was performed in sham-operated mice without aortic banding. Twenty-four $\mathrm{h}$ after ligation, the surviving mice were randomly divided into four groups ( $n=8$ per group): 1 ) sham-operated group, 2) vehicle-treated AB group ('vehicle-treated group'), 3) AB mice treated with AVE 0991 ('AVE 0991 group'). AVE 0991 group was administered orally once a day at AVE $0991(20 \mathrm{mg} \cdot \mathrm{kg} \cdot \operatorname{day}(-1))$ for 4 weeks while isovolumic sodium chloride was administrated in the same manner for the sham-operated and vehicle-treated group [9].

\subsection{Doppler echocardiography}


Chamber dimensions and LV function were measured using transthoracic echocardiography as previously described. Briefly, mice were lightly anesthetized with isoflurane inhalant (1.5-2\% of isoflurane inhalant mixed with $\left.1 \mathrm{~L} / \mathrm{min} 100 \% \mathrm{O}_{2}\right)$. 2D guided M-mode studies were performed using an echocardiographic system (Visual Sonics Vevo 2100 with a 30-MHz transducer). All parameters were averaged for 10 consecutive cardiac cycles and performed by an experienced technician who was blinded to study groups. Averages were used to calculate left ventricular end-diastolic diameter (LVDd), left ventricular posterior wall thickness (LVPWd), ejection fraction (EF), and fractional shortening (FS).

\subsection{Histological analysis}

For the determination of LV hypertrophy, the superior half of the LV tissues were fixed in $10 \%$ neutral buffered formalin and embedded in paraffin. The tissue was sectioned at $4 \mu \mathrm{m}$, rehydrated with ethanol, stained with hematoxylin and eosin, dehydrated, and mounted on slide for light microscopic analysis to evaluate the myocyte diameter. The entire histomorphometric study was performed using a Zeiss-KONTRON IBAS 2.5 Automatic Image Analysis System (Zeiss, Munich, Germany). On each slice, which was characterized according to its location (transmural, epicardial or endocardial) and was subsequently quantified and expressed as the ratio of the scar perimeter $\times 100$ to the sum of external and internal perimeters of the left ventricle. The average of the three slices was calculated. Mean myocyte diameter was defined by 100 cells from sections stained with hematoxylin and eosin [2]. 
The inferior half of LV tissues were rapidly obtained, frozen in liquid nitrogen, and then stored at a $-80^{\circ} \mathrm{C}$ freezer. These tissue samples were used for quantitative real-time PCR (q-PCR) and Western blot.

\subsection{Quantitative real-time PCR ( $q$-PCR)}

Total cellular RNA was isolated using Trizol reagent (Invitrogen). Double-stranded cDNA was synthesized using M-MLV RTase cDNA Synthesis Kit (Takara). Q-PCR was carried out with LightCycler ${ }^{\circledR} 480$ SYBR Green I Master Mix (Roche Diagnostics) in a LightCycler® 480 System. The cycling protocol of PCR reaction used was as follows: $95^{\circ} \mathrm{C}$ for $5 \mathrm{~min}$, followed by 45 cycles of $95^{\circ} \mathrm{C}$ for $10 \mathrm{~s}, 60^{\circ} \mathrm{C}$ for $10 \mathrm{~s}$ and $72{ }^{\circ} \mathrm{C}$ for 20s. The primers used were as followed: ANP, 5'-GTGTCACCCACAACGTGC-3' (forward) and 5'-AGGGCCACATAGCACAGC-3'(reverse); $\quad \beta \quad$-MHC, 5'-GCCCTTTGACCTCAAGAAAG-3', (forward) and 5'-CTTCACAGTCACCGTCTTGC -3' (reverse); NOX2, 5'TTCCAGTGCGTGTTGCTCGAC-3' (forward) and ' GATGGCGGTGTGCAGTGCTAT-3' (reverse); NOX4, 5' GGATCACAGAAGGTCCCTAGCAG-3' (forward) and 5'GCGGCTACATGCACACCTGAGAA-3' (reverse); GAPDH, 5'-ACAGCAACAGGGTGGTGGAC-3' (forward) and 5'-TTTGAGGGTGCAGCGAACTT-3' (reverse) [2].

2.5 Western blot 
Cardiac tissues were homogenized in liquid nitrogen and then in SDS sample buffer to extract total protein. Total protein concentration was determined using the BCA Protein Assay Kit. Thirty micrograms of protein were separated by SDS-PAGE on a $10 \%$ polyacrylamide gel and transferred to a hydrobond-PVDF membrane using a Western blot apparatus. The membrane was incubated with $5 \%$ skim milk in Tris-buffered saline solution with Tween for $2 \mathrm{~h}$ at $4^{\circ} \mathrm{C}$ for blocking nonspecific binding sites. After being washed in Tris-buffered saline solution, the membrane was incubated with primary antibodies for NOX2 NOX4 or $\beta$-actin (Santa Cruz Biochemicals; $1: 1000$ ) overnight at $4^{\circ} \mathrm{C}$. Horseradish peroxidase-conjugated $\mathrm{IgG}$ was used as a secondary antibody (Santa Cruz Biochemicals). The protein expression levels were determined by analyzing the signals captured on the PVDF membranes using an image analyzer (Las-1000, Fuji-film; Ref.) [2].

\subsection{Statistical analysis}

All values were expressed as mean \pm standard error (SE). The statistical analysis on differences among the groups was done by one-way ANOVA. It was followed by Newnam Keul's for multiple comparisons. Differences were judged to be significant when $P<0.05$. Statistical analyses were performed using SPSS 10.0 statistics software (SPSS Inc., Chicago, IL, USA).

\section{Results}

\subsection{AVE0991 treatment attenuates cardiac hypertrophy in post-AB mice}

To further validate the finding at a cellular level, the histological analysis of the LV tissue was performed and cardiac myocyte diameter was measured. As expected, 
cardiac hypertrophy indices, including left ventricular weight, left ventricular weight /body weight ratio, mean myocyte diameter as well as expression of the hypertrophic markers $\mathrm{ANP}$ and $\beta$-MHC were significantly higher in the vehicle-treated $\mathrm{AB}$ group than in the sham-operated group 4 weeks after operation $(\mathrm{P}<0.05)$. Treatment with AVE0991 further reduced cardiac hypertrophy compared to vehicle-treated AB group (Table, Fig.1-2).

\subsection{AVE0991 treatment improves systolic function in post-AB mice}

To further investigate whether treatment with AVE0991 improved cardiac functional recovery of post- $\mathrm{AB}$, cardiac function was measured in the sham-control, post- $\mathrm{AB}$, AVE0991 treated post-AB mice. After 28 days of $A B$, post-AB mice had a significant lower ejection fraction (EF), and fractional shortening (FS) and a higher left ventricular end-diastolic diameter (LVDd) compared to sham-control. Treatment of post-MI mice with AVE0991 resulted in a significant decrease in LVDd and a significantly increase in EF and FS compared to post-AB group. AVE0991 had no effect on Left ventricular posterior wall thickness (LVPWd) (Table, Fig.3).

\subsection{The mRNA and protein expression of NOX2 and NOX4 is increased in} AVE0991 treated post-AB mice

To test whether AVE0991 regulated oxidative stress function, the mRNA and protein expression of NOX2 and NOX4 was examined in myocardial tissue of post-AB mice. As shown in Fig 3, the mRNA expression of NOX2 and NOX4 was significantly higher in the vehicle-treated group compared with the sham-operated group $(\mathrm{P}<0.01)$. 
Similarly, the levels of NOX2 and NOX4 protein expression were significantly upregulated in post- $\mathrm{AB}$ mice. That is mean that the levels of oxidative stress were significantly elevated in the vehicle-treated group compared with the sham-operated group $(\mathrm{P}<0.01)$. Moreover, AVE 0991 treatment suppressed this increase in NOX 2 and NOX 4 mRNA and protein expression, meaning that AVE 0991 reduced oxidative stress of cardiac hypertrophy mice (Table, Fig.4).

\section{Discussion}

Several studies demonstrated that ANG-(1-7) has antihypertrophic and antifibrotic functions. AVE 0991 is the agonist of Ang-(1-7)'s receptor, previous evidence showed that AVE 0991 attenuated cardiac remodeling in renovascular hypertensive rats $[8,11]$. The present study suggested that AVE 0991 treatment decreased cardiac hypertrophy induced by aortic banding and improved heart function. AVE 0991 treatment may be represent a promising therapy for prevent cardiac hypertrophy and progression to heart failure.

Ang-(1-7) is a biologically active member of the renin-angiotensin system (RAS), which inhibits many detrimental cardiovascular disease phenotypes and holds cardioprotective actions [13-15]. Substantial evidence suggests Ang-(1-7) treatment ameliorated myocardial hypertrophy and fibrosis through receptor Mas [13]. Recent data have demonstrated that the nonpeptide AVE-0991 was a selective ligand for the Ang-(1-7) receptor Mas and had actions similar to ANG-(1-7) [11]. Most recently we reported that AVE 0991 treatment ameliorated cardiomyocyte hypertrophy [7]. We 
suggest that AVE 0991 treatment could attenuate cardiac hypertrophy induced by pressure overload. In this study, aortic banding mice were used as a model of physiological cardiac hypertrophy. As expected, treatment with AVE 0991 reduced cardiac hypertrophy indices which significantly increased in vehicle-treated AB group, including left ventricular weight, left ventricular weight /body weight ratio, mean myocyte diameter as well as expression of the hypertrophic markers ANP and $\beta$ -MHC. It means that AVE 0991 treatment attenuates cardiac hypertrophy induced by aortic banding. Furthermore, treatment with AVE 0991 improved cardiac function. We show that AVE 0991 treatement prevented the increases parameters in aortic banding mice. Beneficial changes included improvement in LVDd, EF, and FS, which is consistent with the previous study $[8,10-11]$.

How does AVE 0991 affect cardiac hypertrophy and cardiac function after aortic banding? Previous studies indicated that oxidative stress plays a crucial role in the process of cardiac hypertrophy. Oxidative stress describes an imbalance between antioxidant defences and the production of reactive oxygen species (ROS). Several evidences showed that ROS plays an important role in the pathophysiology of heart failure and Ang II-induced cardiovascular disease. Nicotinamide-adenine dinucleotide phosphate (NADPH) oxidases are key enzymes that affect ROS production and are involved in both physiological and pathological processes in cells. NOX2 and NOX4 are the major isoforms of NADPH oxidase in the heart. Previous studies have implicated the involvement of NOXes in cardiac hypertrophy [15-18]. Zhang et al [18] reported that NOX4 mediates cardiac hypertrophy and cell death. In the present study, 
we showed that expression of NOX2 and NOX4 in myocardial tissues was significantly higher in post-AB mice. That is meaning that the levels of oxidative stress were significantly elevated in cardiac hypertrophy mice, which is consistent with the previous study [19]. Furthermore, Raffai has reported that Ang-(1-7) and AVE 0991 constitutes a potential therapeutic strategy to alleviate NOX-mediated oxidative stress [12]. As expected, our results showed that NOX2 and NOX4 mRNA and protein expression levels in myocardial tissues were significantly lower in AVE 0991-treated mice compared with vehicle-treated mice, suggesting that AVE 0991 treatment prevents cardiac hypertrophy and improves cardiac function may be through reducing oxidative stress levels.

In conclusion, our results suggest that sustained upregulation of oxidative stress contributes to cardiac hypertrophy and cardiac dysfunction after aortic banding. AVE 0991 treatment could prevent ventricular hypertrophy and cardiac dysfunction, a process that may be associated with the inhibition of oxidative stress.

\section{Acknowledgments}

This work was supported by the National Science Foundation of China [Grants 81100171] [Grants 81270318] [Grants 81200173], the Science and technology program of Guangdong Province (grant No. 2012B031800296) and the Specialized Research Fund for the Doctoral Program of Higher Education (Grant No. 20120171120078). 


\section{References Note:}

[1] Oka T, Akazawa H, Naito AT, et al. Angiogenesis and cardiac hypertrophy: maintenance of cardiac function and causative roles in heart failure. Circ. Res. 114 (2014) 565-571.

[2] He JG, Chen YL, Chen BL, et al. B-type natriuretic peptide attenuates cardiac hypertrophy via transforming growth factor- $\beta 1 / \mathrm{smad} 7$ pathway in vivo and in vitro. Clin. Exp. Pharmacol. Physiol. 37 (2010) 283-289.

[3] Liu C, Xue R, Wu D, et al. REDD1 attenuates cardiac hypertrophy via enhancing autophagy. Biochem Biophys Res Commun. 454 (2014) 215-220.

[4] Hahn NE, Musters RJ, Fritz JM, et al. Early NADPH oxidase-2 activation is crucial in phenylephrine-induced hypertrophy of H9c2 cells. Cell Signal. 26 (2014) 1818-1824.

[5] Rizzi E, Ceron CS, Guimaraes DA, et al. Temporal changes in cardiac matrix metalloproteinase activity, oxidative stress, and TGF- $\beta$ in renovascular hypertension-induced cardiac hypertrophy. Exp. Mol. Pathol. 94 (2013) 1-9.

[6] Liu M, Ye J, Gao S, et al. Salvianolic acid B protects cardiomyocytes from angiotensin II-induced hypertrophy via inhibition of PARP-1. Biochem Biophys Res Commun. 444 (2014) 346-353.

[7] He JG, Chen SL, Huang YY, et al.The nonpeptide AVE0991 attenuates myocardial hypertrophy as induced by angiotensin II through downregulation of transforming growth factor-beta1/Smad2 expression. Heart Vessels. 25 (2010) 438-443.

[8] Ferreira AJ, Oliveira TL, Castro MC, et al. Isoproterenol-induced impairment of heart function and remodeling are attenuated by the nonpeptide angiotensin-(1-7) analogue AVE 0991. Life Sci. 81 (2007) 916-923. 
[9] Linda E, Frank S, Melanie S, et al. The angiotensin-(1-7) receptor agonist AVE0991 is cardioprotective in diabetic rats. Eur J Pharmacol. 590 (2008) 276-280.

[10] Zeng WT, Chen WY, Leng XY, et al.Impairment of cardiac function and remodeling induced by myocardial infarction in rats are attenuated by the nonpeptide angiotensin-(1-7) analog AVE 0991. Cardiovasc. Ther. 30 (2012) 152-161.

[11] Cunha TM, Lima WG, Silva ME, et al. The nonpeptide ANG-(1-7) mimic AVE 0991 attenuates cardiac remodeling and improves baroreflex sensitivity in renovascular hypertensive rats. Life Sci. 92 (2013) 266-275.

[12] Raffai G, Durand MJ, Lombard JH. Acute and chronic angiotensin-(1-7) restores vasodilation and reduces oxidative stress in mesenteric arteries of salt-fed rats. Am. J. Physiol. Heart Circ. Physiol. 301 (2011) H1341- H1352.

[13] Mori J, Patel VB, Abo Alrob O, et al. Angiotensin 1-7 ameliorates diabetic cardiomyopathy and diastolic dysfunction in $\mathrm{db} / \mathrm{db}$ mice by reducing lipotoxicity and inflammation. Circ. Heart Fail. 7 (2014) 327-339.

[14] Magierowski M, Jasnos K, Pawlik M, et al. Role of angiotensin-(1-7) in gastroprotection against stress-induced ulcerogenesis. The involvement of mas receptor, nitric oxide, prostaglandins, and sensory neuropeptides. J. Pharmacol. Exp. Ther. 347 (2013) 717-726.

[15] Kuroda J, Pain J, Fu C, et al. Upregulation of Nox4 by hypertrophic stimuli promotes apoptosis and mitochondrial dysfunction in cardiac myocytes. Circ. Res. 106 (2010) $1253-1264$ 
[16] Kuroda J, Ago T, Matsushima S, et al. NADPH oxidase 4 (Nox4) is a major source of oxidative stress in the failing heart. Proc. Natl. Acad. Sci. USA. 107 (2010) 15565-15570.

[17] Grieve DJ, Byrne JA, Siva A, et al. Involvement of the nicotinamide adenosine dinucleotide phosphate oxidase isoform Nox 2 in cardiac contractile dysfunction occurring in response to pressure overload. J. Am. Coll. Cardiol. 47 (2006) 817-826.

[18] Zhang M, Brewer AC, Schröder K, et al. NADPH oxidase-4 mediates protection against chronic load-induced stress in mouse hearts by enhancing angiogenesis. Proc. Natl. Acad. Sci. USA. 107 (2010) 18121-18126.

[19] Gwathmey TM, Pendergrass KD, Reid SD, et al. Angiotensin-(1-7)-angiotensin-converting enzyme 2 attenuates reactive oxygen species formation to angiotensin II within the cell nucleus. Hypertension 55 (2010) 166-171. 


\section{Figure legend:}

Fig1. Mean myocyte diameter in 4 weeks after aortic banding Representative hematoxylin and eosin-stained high magnification (X400) of left ventricles from different groups. A: sham-operated group $(n=8), B$ : vehicle-treated group $(n=8), C$ : AVE 0991 group $(n=8)$.

Fig2. Expression of ANP and $\boldsymbol{\beta}$-MHC mRNA Sham: sham-operated group ( $\mathrm{n}=8)$, AB: vehicle-treated group ( $\mathrm{n}=8)$, AVE 0991: AVE 0991 group $(\mathrm{n}=8) . *$ : $P<0.01$ vs sham-operated group, $\star: P<0.01$ vs vehicle-treated group.

Fig3. Echocardiographic assessment 4 weeks after aortic banding A:

sham-operated group (n=8), B: vehicle-treated group (n=8), C: AVE 0991 group $(n=8)$.

Fig4. Expression of NOX2 and NOX4 mRNA and protein Sham: sham-operated group (n=8), AB: vehicle-treated group ( $\mathrm{n}=8)$, AVE 0991: AVE 0991 group $(n=8)$. *: $P<0.05$ vs sham-operated group, $\star: P<0.05$ vs vehicle-treated group. 
Table 1: Echocardiography and hemodynamic measurements 4 weeks after aortic banding.

\begin{tabular}{lccc}
\hline Parameters & $\begin{array}{l}\text { Sham group } \\
(\mathrm{n}=8)\end{array}$ & $\begin{array}{c}\text { Vehicle-treated group } \\
(\mathrm{n}=8)\end{array}$ & $\begin{array}{c}\text { AVE0991 group } \\
(\mathrm{n}=8)\end{array}$ \\
\hline Wt $(\mathrm{g})$ & $28.44 \pm 0.50$ & $28.15 \pm 0.44$ & $27.68 \pm 0.42$ \\
LVW (mg) & $159.05 \pm 14.62$ & $222.29 \pm 7.55^{\mathrm{b}}$ & $159.61 \pm 6.78^{\mathrm{d}}$ \\
LVMI (mg/g) & $5.57 \pm 0.47$ & $7.89 \pm 0.24^{\mathrm{b}}$ & $5.83 \pm 0.21^{\mathrm{d}}$ \\
MMD (um) & $12.75 \pm 0.35$ & $15.46 \pm 0.21^{\mathrm{b}}$ & $13.53 \pm 0.56^{\mathrm{d}}$ \\
EF (\%) & $61.73 \pm 3.87$ & $41.82 \pm 5.58^{\mathrm{a}}$ & $58.16 \pm 2.78^{\mathrm{c}}$ \\
FS (\%) & $32.57 \pm 2.65$ & $21.65 \pm 3.26^{\mathrm{a}}$ & $30.00 \pm 1.29$ \\
LVDd (mm) & $3.26 \pm 0.18$ & $4.32 \pm 0.20^{\mathrm{b}}$ & $3.48 \pm 0.19^{\mathrm{c}}$ \\
LVPWd (mm) & $0.99 \pm 0.05$ & $1.13 \pm 0.06$ & $1.06 \pm 0.04$ \\
\hline
\end{tabular}

${ }^{\mathrm{a}} P<0.05$ vs. sham-operated group, ${ }^{\mathrm{b}} P<0.01$ vs. sham-operated group, ${ }^{\mathrm{c}} P<0.05$ vs. vehicle-treated group, and ${ }^{\mathrm{d}} P<0.01$ vs. vehicle-treated group. Wt: body weight, LVW: left ventricular weight, LVMI: left ventricular weight/body weight ratio, MMD: mean myocyte diameter, EF: ejection fraction, FS: fractional shortening, LVDd: left ventricular end-diastolic diameter, and LVPWd: left ventricular posterior wall thickness. 
Fig. 1
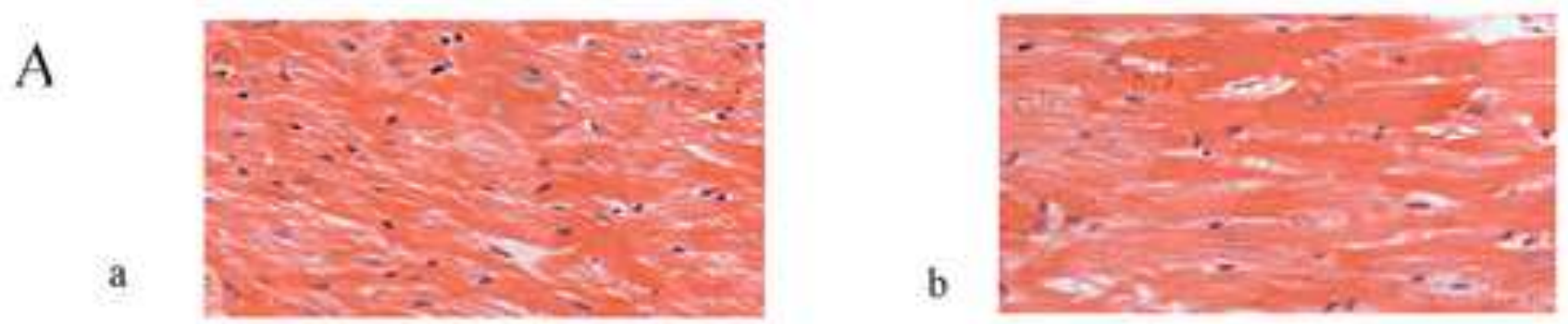

c

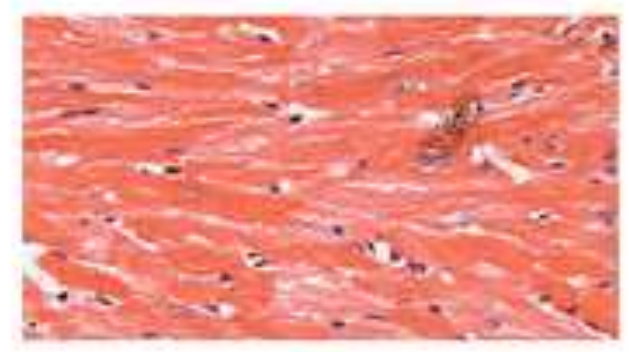

B
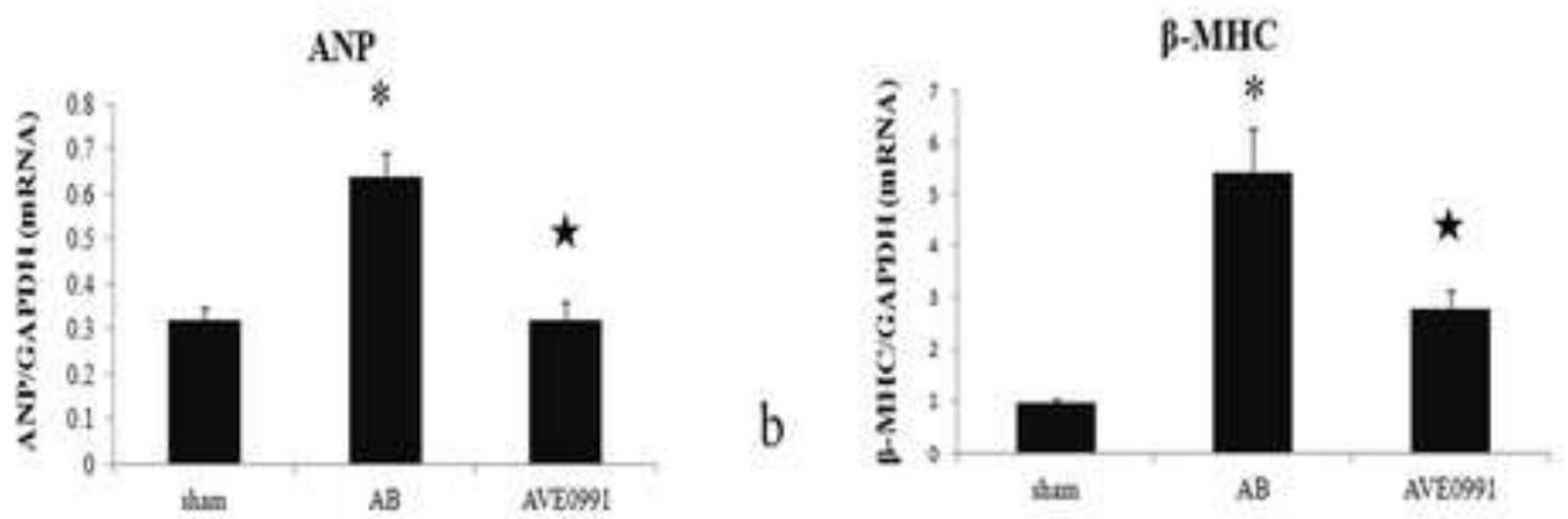

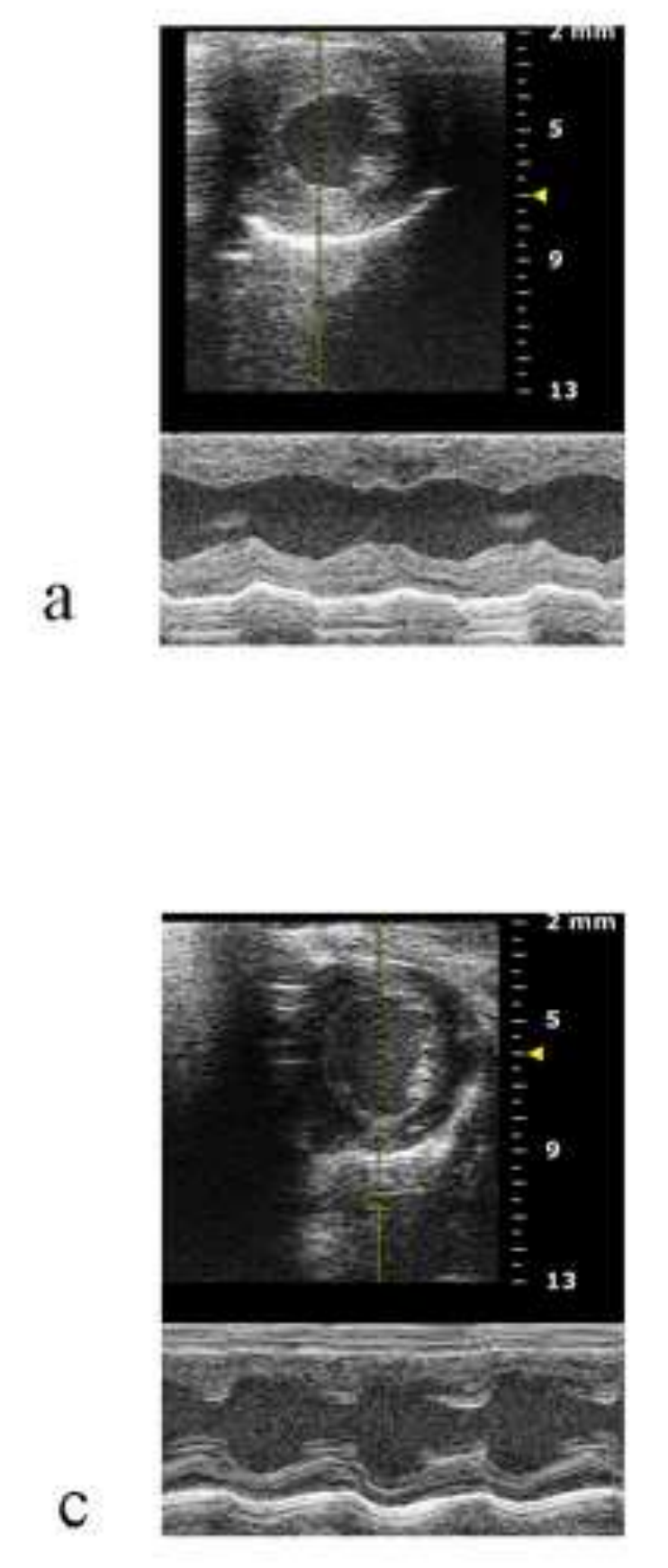

Figure 2

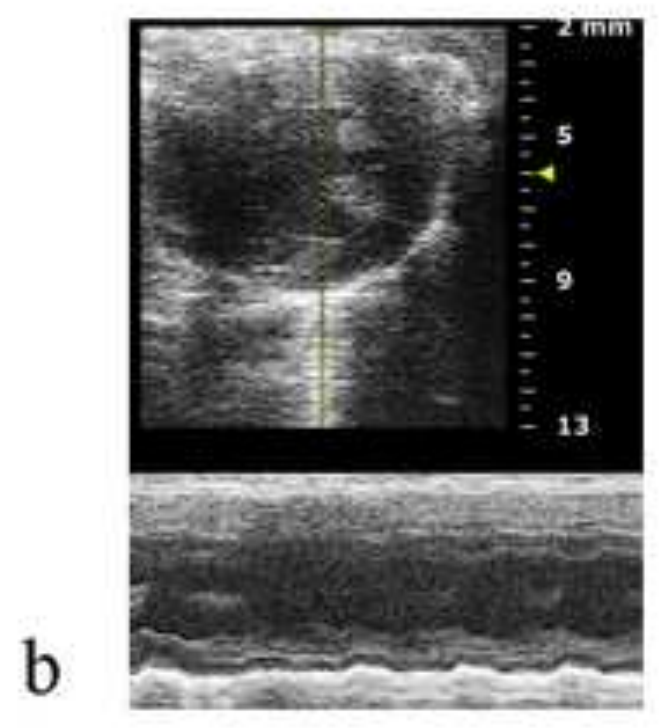

.

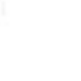

\section{Fig. 2}


Fig. 3
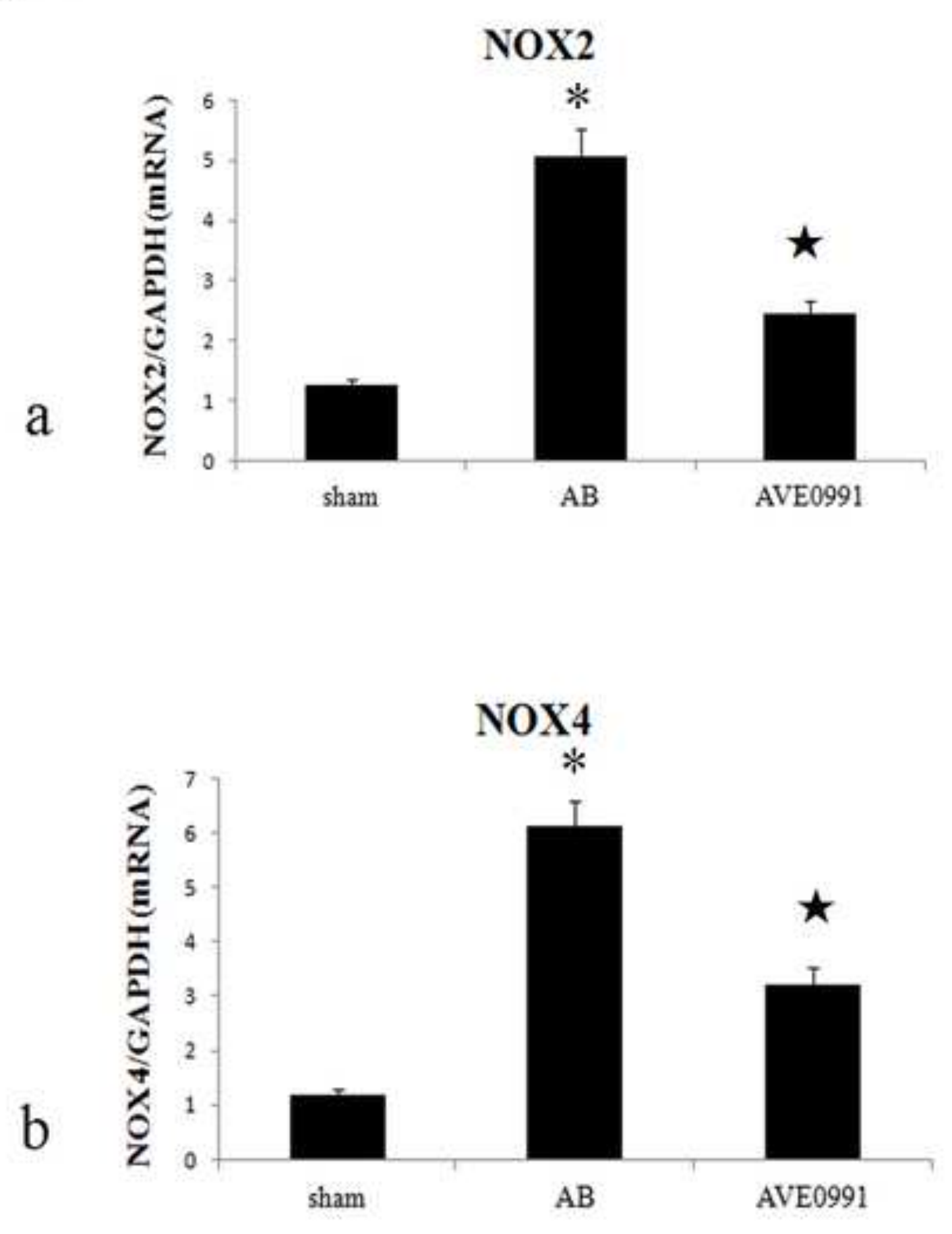

Figure 3

NOX4

Fig.

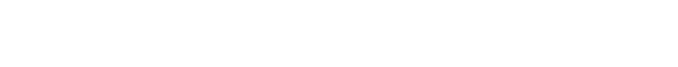

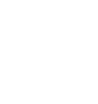

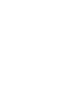

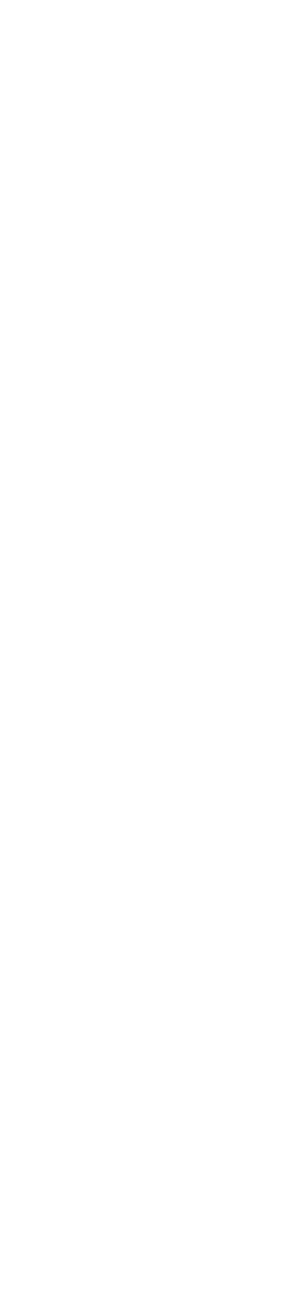

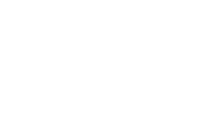

Nox 2

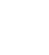

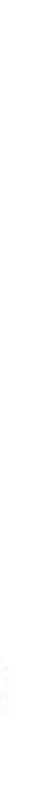


Fig. 4
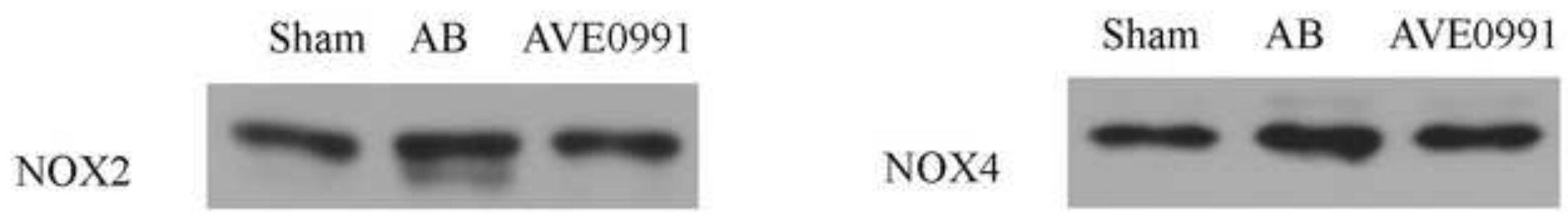

a

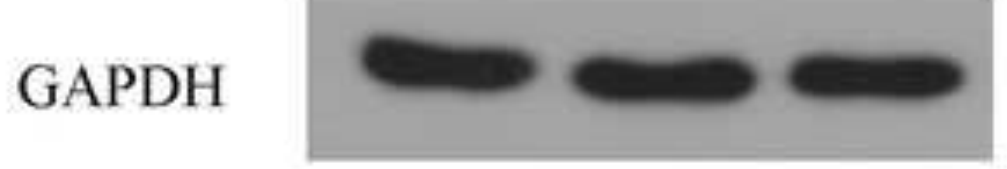

GAPDH

NOX2
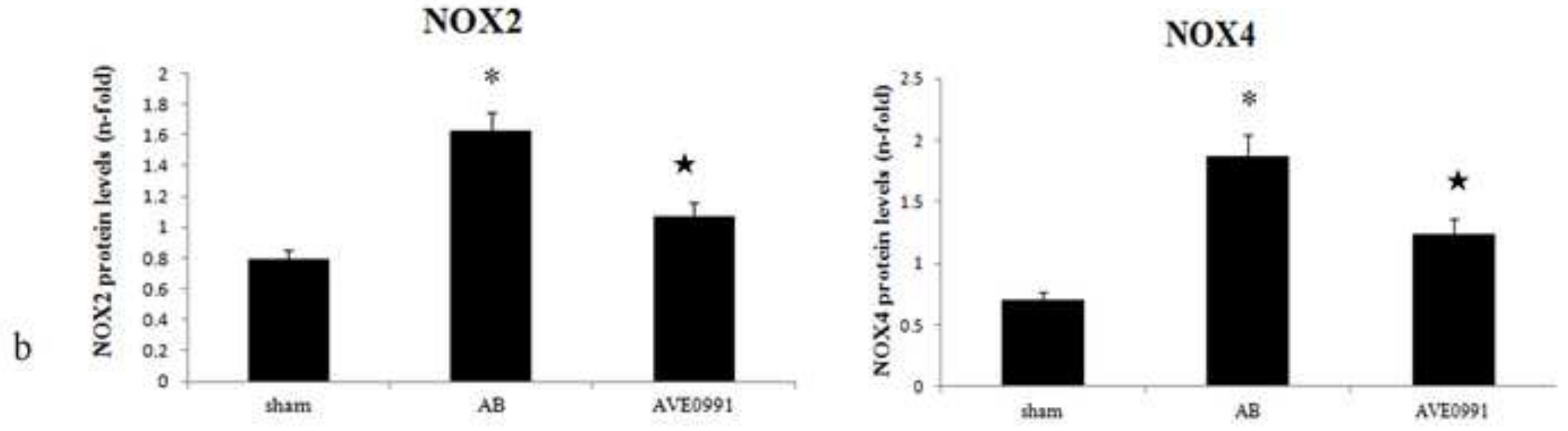